OPEN ACCESS

Edited by:

Tzi Bun Ng,

The Chinese University of Hong Kong,

China

Reviewed by:

Ricardo Martinez,

Eli Lilly, United States

Weicheng Liang,

Sun Yat-Sen University, China

*Correspondence:

Xu Che

drchexu@126.com

$\operatorname{Lin} X u$

drxulin@126.com

${ }^{\dagger}$ These authors have contributed equally to this work

Specialty section:

This article was submitted to

Pharmacology of Anti-Cancer Drugs,

a section of the journal

Frontiers in Oncology

Received: 24 July 2020

Accepted: 08 April 2021

Published: 29 April 2021

Citation:

Huang X, Wang C, Ma T, Huang Z,

Zhou H, Xu L, Zhang R, Zhao J,

Zhang $Y$, Huang $Z$, Shao $L$, Wang $Y$, Yang $F$ and Che $X$ (2021)

The Efficacy of Combined Cisplatin and Nanoparticle Albumin-Bound Paclitaxel in a Stage IV Pancreatic Squamous Cell Carcinoma Patient

With a Somatic BRCA2 Mutation: A Case Report.

Front. Oncol. 11:585983. doi: 10.3389/fonc.2021.585983

\section{The Efficacy of Combined Cisplatin and Nanoparticle Albumin-Bound Paclitaxel in a Stage IV Pancreatic Squamous Cell Carcinoma Patient With a Somatic BRCA2 Mutation: A Case Report}

Xiaozhun Huang ${ }^{1+}$, Chunling Wang ${ }^{2 \dagger}$, Teng $\mathrm{Ma}^{1}$, Zhangkan Huang ${ }^{1}$, Houhong Zhou ${ }^{1}$, Lin Xu ${ }^{1 *}$, Renjie Zhang ${ }^{1}$, Jianjun Zhao ${ }^{1}$, Yefan Zhang ${ }^{1}$, Zhen Huang ${ }^{1}$, Lin Shao ${ }^{3}$, Yang Wang ${ }^{3}$, Fan Yang ${ }^{3}$ and Xu Che ${ }^{1,4 *}$

\footnotetext{
${ }^{1}$ Department of Hepatobiliary Surgery, National Cancer Center/National Clinical Research Center for Cancer/Cancer Hospital and Shenzhen Hospital, Chinese Academy of Medical Sciences and Peking Union Medical College, Shenzhen, China, 2 Department of Hospital Acquired Infection Control, National Cancer Center/National Clinical Research Center for Cancer/ Cancer Hospital and Shenzhen Hospital, Chinese Academy of Medical Sciences and Peking Union Medical College, Shenzhen, China, ${ }^{3}$ Department of Medicine, Burning Rock Biotech, Guangzhou, China, ${ }^{4}$ Department of Gastrointestinal and Pancreatic Surgery, National Cancer Center/National Clinical Research Center for Cancer/Cancer Hospital, Chinese Academy of Medical Sciences and Peking Union Medical College, Beijing, China
}

Pancreatic squamous cell carcinoma (SCC) is a rare primary pancreatic malignancy with a poor prognosis. The median overall survival (OS) for metastatic setting is only 4 months and the optimal management remains poorly defined. In the present study, we report a 52-year-old female patient with stage IV primary SCC of the pancreas harboring a deleteous BRCA2 somatic mutation. After 10 cycles of chemotherapy of cisplatin combined with nanoparticle albumin-bound paclitaxel, metastatic lesions in the liver and lymph nodes achieved radiographic complete responses and pancreatic lesion shrank from 5.7 to $1.5 \mathrm{~cm}$ in diameter. The patient subsequently underwent a posterior radical antegrade modular pancreatosplenectomy with $\mathrm{RO}$ resection and residual liver lesions were also resected. After 3 months, a tumor relapsed in the liver. She was then treated with olaparib combined with pembrolizumab and achieved stable disease on the liver lesion. The patient eventually died from cerebral hemorrhage with a long OS of 21 months. Our case demonstrated a favorable clinical activity and survival advantage of the combined cisplatin and nanoparticle albumin-bound paclitaxel, which might serve as a therapeutic option for the patient with BRCA-mutant pancreatic SCC.

Keywords: pancreatic squamous cell carcinoma, cisplatin, nanoparticle albumin-bound paclitaxel, BRCA2 mutation, overall survival 


\section{INTRODUCTION}

Pancreatic squamous cell carcinoma (SCC) is a rare primary pancreatic malignancy, comprising $0.5-5 \%$ of all exocrine pancreatic neoplasms. It is characterized by worse survival, largely attributable to that the majority of patients with this entity present with stage IV disease at initial diagnosis (1). The median overall survival (OS) has been reported to range from 7 to 14 months (2-4). In addition, the optimal management for pancreatic SCC remains poorly defined. The initial choice of chemotherapy is extrapolated from experience of pancreatic adenocarcinoma, to which patients often respond poorly (5).

In recent years, greater insight into the molecular biology of pancreatic cancer has prompted new treatment strategies. BRCA genes code for proteins that are involved in homologous recombination repair of DNA double-strand breaks (6). Approximately $10 \%$ of pancreatic cancers are associated with germline or somatic mutation in BRCA1 or BRCA2 (7). Clinical evidence has shown the efficacy and survival advantage of drugs targeting the DNA repair pathway such as platinum-based chemotherapy and PARP inhibitors in germline BRCA1/2mutant patients with pancreatic adenocarcinoma $(1,8)$. However, little is known about the efficacy of these drugs in $B R C A$-mutant pancreatic SCC owing to its rarity.

Herein, we present a 52-year-old female patient with a stage IV pancreatic SCC harboring a somatic BRCA2 deleterious mutation, who responded favorably to the chemotherapy of cisplatin and nanoparticle albumin-bound paclitaxel, and achieved a long OS of 21 months.

\section{CASE DESCRIPTION}

The patient's clinical course was illustrated in Figure 1A. A 52year-old female patient presented at the hospital in June, 2018 in a good physical condition, complaining of pain in the middle and upper abdomen accompanied by acid reflux and heartburn over the past 5 months. Her serum CA19.9, SCC, and CEA levels were $65.82 \mathrm{U} / \mathrm{ml}, 25.5 \mathrm{ng} / \mathrm{ml}$, and $11.65 \mathrm{ng} / \mathrm{ml}$, respectively. An abdomen computed tomography $(\mathrm{CT})$ indicated a tumor measuring $7 \times 6 \mathrm{~cm}$ located in the tail of the pancreas that invaded the posterior wall of the stomach and the splenic artery, accompanied by multiple hepatic and lymph node metastases in the left supraclavicular fossa (Figure 1B). Subsequently an endoscopic ultrasound (EUS)-guided biopsy was performed on the pancreatic lesion and the histological and immunohistochemically tests confirmed a cT4N1M1 primary pure squamous cell carcinoma (Figure 2A).

A sequencing performed on the biopsied specimen revealed a stop-gain mutation in BRCA2 exon 11 (c.1830A>T, p.K944*) (Figure S1), which has been reported as a pathogenic germline mutation. In addition, the patient had a tumor mutational burden (TMB) of 5.4 mut/Mb and a stable microsatellite status (MSS). Based on the genetic results, a regimen of nanoparticle albumin-bound paclitaxel $\left(125 \mathrm{mg} / \mathrm{m}^{2}\right)$ combined with cisplatin $\left(75 \mathrm{mg} / \mathrm{m}^{2}\right)$ was eventually decided after a multi-disciplinary panel discussion.

The combined chemotherapy was initiated in July, 2018 (Figure 1A). After five cycles of treatment, the patient achieved partial response (PR) on primary pancreatic and metastatic lesions revealed by a total abdomen CT scan

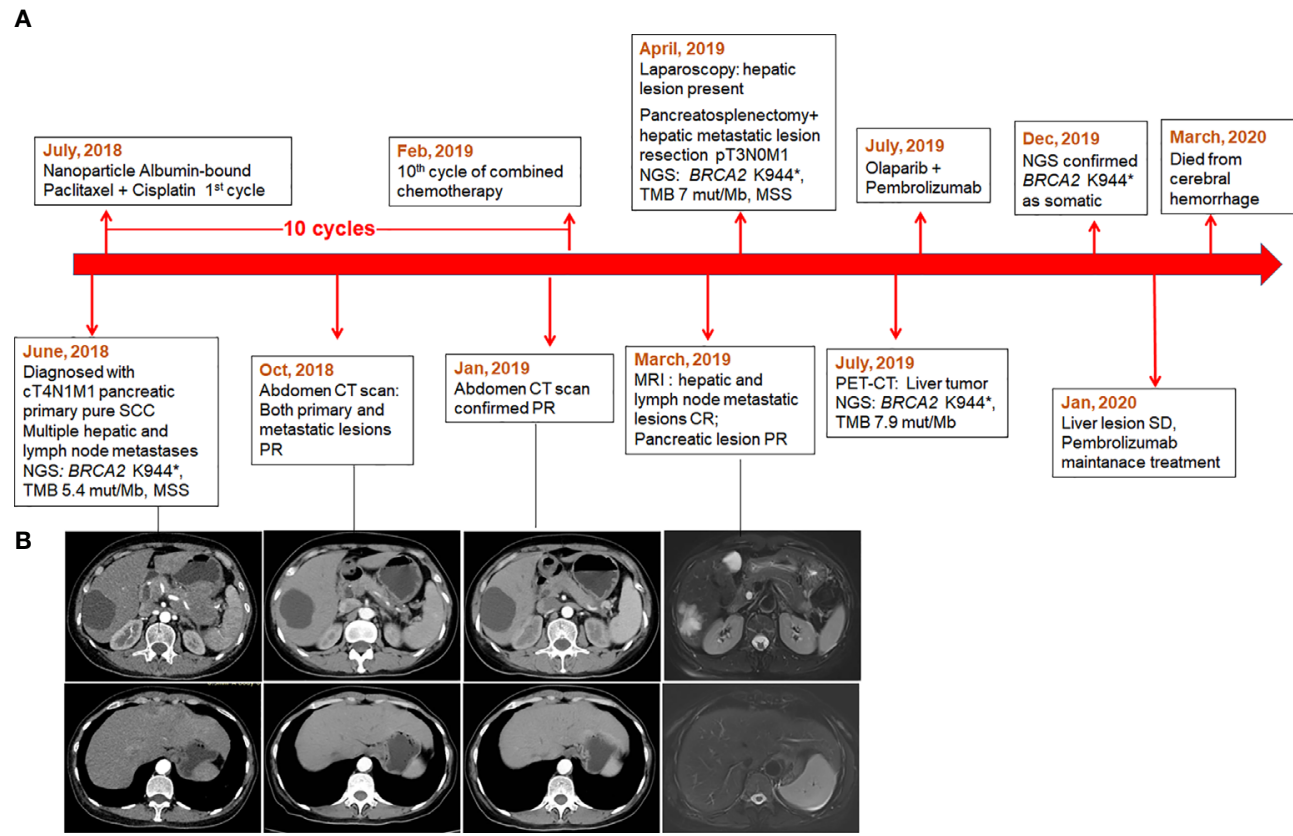

FIGURE 1 | The timeline of patient's treatment history and the response of the tumor lesions. (A) Time line; (B) The radiographic imaging of pancreatic and metastatic liver lesions. 


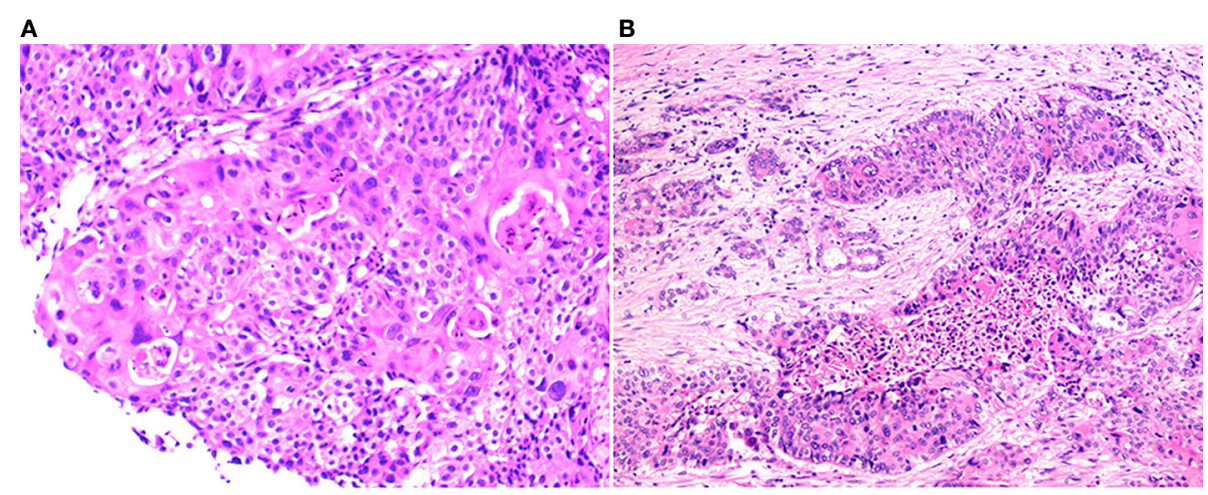

FIGURE 2 | Hematoxylin and eosin (HE) stain of the pancreaticlesion. (A) The biopsy of the pancreatic lesion for initial diagnosis indicated squamous cell carcinoma (200x). (B) The HE stain of the surgical sample after the combined chemotherapy (100x).

(Figure 1B) and the serum biomarkers (CA19.9, SCC, and CEA) declined into a normal range. She remained as PR after eight cycles. A magnetic resonance imaging (MRI) was performed in March, 2019 after 10 cycles of combined chemotherapy and showed that both hepatic and lymph node metastases (regional and distanced lymph nodes) achieved complete response (CR) and the primary pancreatic lesion shrank from the initial 5.7 to $1.5 \mathrm{~cm}$ in diameter (Figure 1B).

Since the imaging examination indicated tumor downstaging, which might be qualified for surgical resection, a preoperative laparoscopy was conducted in April, 2019, and detected multiple nodules on the liver surface. No hepatic space-occupying lesion was detected by the intraoperative US. The nodules on hepatic segments 2, 3, and 4 were resected and sent for histopathological test, and results showed a few malignant tumor cells in the S2 nodule. A laparotomy continued under the request of the patient's family and revealed a lesion of $4 \times 3 \mathrm{~cm}$ in the pancreatic tail and multiple hepatic lesions. Subsequently, a posterior radical antegrade modular pancreatosplenectomy was performed and hepatic metastatic lesions were also resected. Histological examination of the resected specimens revealed severe tumor degeneration after the combined chemotherapy (Figure 2B) with vital tumor cells present only in small areas of primary and liver metastatic lesions. Two of the four peripancreatic lymph nodes had severe degeneration; no residual tumor cells were found in lymph nodes $(n=12)$. The pathological classification of pT3N0M1 was defined. The sequencing on the tumor tissue showed the retaining of

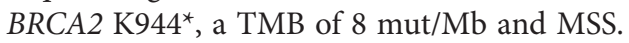

The patient experienced postoperative thrombocytopenia (platelet account: $6-9 \times 10^{9} / \mathrm{L}$ ) of unknown cause and the platelet account returned to normal $\left(50-60 \times 10^{9} / \mathrm{L}\right) 3$ months later without any treatment. A repeat PET-CT in July, 2019 showed a liver recurrent lesion of $3.3 \mathrm{~cm}$ in diameter. The patient was then treated with olaparib (200 mg per day) combined with pembrolizumab (100 mg per 21 days) and achieved stable disease (SD) on her liver lesion 6 months later. The plasma and matched white blood cell samples of the patient were sent for sequencing using a panel with 520-cancer-related genes. The results confirmed BRCA2 K944* as a somatic mutation, and showed a TMB of 7.98 mut/Mb and MSS status. The patient experienced hepatic artery embolization and severe low platelet count. She died from a cerebral hemorrhage in March, 2020 with an OS of 21 months.

\section{DISCUSSION}

Surgery remains the cornerstone in the management of pancreatic SCC. However, the standard care for the metastatic setting is still lacking $(9,10)$. Data from sporadic reports revealed that $38.5 \%$ of metastatic patients received palliative care, and others commonly underwent a combination of cisplatin and 5fluorouracil or gemcitabine, showing a short median OS of 4 months, compared with 17 months in the resectable group and 8 months in the locally advanced tumor group (11). A pool of data regarding pancreatic SCC available in the public domain showed a median OS of 7 months in 54 patients with pancreatic SCC, and resectable cases had significantly better OS when compared with non-resectable cases (10 months versus 4 months, respectively) (10). In stage IV disease, a significant difference in median OS was noticed between the patients who received chemoradiation (4.9 months) as compared to that of the patients who did not receive chemoradiation (1.5 months) (12). S. Kumar Das Majumdar et al. reported a case of a locally advanced SCC of the pancreatic tail showing an OS of 1 year following treatment with an albumin-free nanosomal paclitaxel lipid suspensionbased regimen (13). A recent study described a patient with T2N0M0R0 primary SCC of the pancreas, who received chemotherapy of gemcitabine following surgery and achieved a long relapse-free survival of 26 months (11).

In the present study, the combined chemotherapy of cisplatin and nanoparticle albumin-bound paclitaxel showed a substantial down-sizing effect in a BRCA2-mutant patient with stage IV pancreatic SCC, increased the tumor resectability and 
significantly prolonged the OS to 21 months, which is the longest survival reported for metastatic pancreatic SCC so far.

NCCN guidance recommends cisplatin combined with gemcitabine for patients with locally advanced or metastatic pancreatic adenocarcinoma for known BRCA1/2 mutation. However, the BRCA mutation in pancreatic SCC has rarely been reported. Schultheis et al. described a locally advanced pancreatic SCC patient with a germline BRCA2 mutation, who responded favorably to neoadjuvant radiochemotherapy with gemcitabine and obtained an OS of 10 months (14). To the best of our knowledge, our study presents the first BRACdeficient pancreatic SCC case with a beneficial response to cisplatin-based chemotherapy. In addition, the nanoparticle albumin-bound paclitaxel has been shown to reduce the dense tumor stroma, allowing the chemotherapeutics being delivered to tumor tissue more efficiently (15), which might further explain the tremendously well tumor response in our case.

Despite the preoperative laparoscopy revealed liver metastatic lesions after the combined chemotherapy, the patient underwent radical resection requested by her family and $\mathrm{R} 0$ resection was achieved. Postoperatively, she received a regimen of olaparib combined with pembrolizumab after the liver lesion relapsed, and achieved an SD, which suggests a potential efficacy of PARP inhibitors in somatic BRAC1/2 mutant pancreatic SCC. Although olaparib is only approved for pancreatic adenocarcinoma with germline BRCA1/2 mutation, the latest study showed comparable response rates and survivals with PARP inhibitors for patients harboring somatic versus germline BRCA mutations (16). Our study also provides preliminary evidence for extending the indication to the somatic $B R A C 1 / 2$ mutant setting.

In conclusion, we described an exceptional response and survival advantage in a BRCA2-mutant patient with metastatic pancreatic SCC who was treated with the combination of cisplatin and nanoparticle albumin-bound paclitaxel. Our data suggest an effective and tolerable therapeutic option for this rare disease entity.

\section{REFERENCES}

1. Golan T, Kanji ZS, Epelbaum R, Devaud N, Dagan E, Holter S, et al. Overall Survival and Clinical Characteristics of Pancreatic Cancer in BRCA Mutation Carriers. Br J Cancer (2014) 111(6):1132-8. doi: 10.1038/ bjc. 2014.418

2. Brijbassie A, Stelow E, Shami VM. Squamous Cell Carcinoma of the Pancreas: A Case Report and Review of Literature. Gastroenterol Res (2014) 7(3-4):1024. doi: 10.14740/gr605w

3. Serafini F, Rosemurgy AS2, Carey LC. Squamous Cell Carcinoma of the Pancreas. Am J Gastroenterol (1996) 91(12):2621-2.

4. Brown HA, Dotto J, Robert M, Salem RR. Squamous Cell Carcinoma of the Pancreas. J Clin Gastroenterol (2005) 39(10):915-9. doi: 10.1097/ 01.mcg.0000180636.74387.e6

5. Makarova-Rusher OV, Ulahannan S, Greten TF, Duffy A. Pancreatic Squamous Cell Carcinoma: A Population-Based Study of Epidemiology, Clinicopathologic Characteristics and Outcomes. Pancreas (2016) 45 (10):1432-7. doi: 10.1097/MPA.0000000000000658

6. Walsh CS. Two Decades Beyond BRCA1/2: Homologous Recombination, Hereditary Cancer Risk and a Target for Ovarian Cancer Therapy. Gynecol Oncol (2015) 137(2):343-50. doi: 10.1016/j.ygyno.2015.02.017

\section{DATA AVAILABILITY STATEMENT}

The original contributions presented in the study are included in the article/Supplementary Material. Further inquiries can be directed to the corresponding authors.

\section{ETHICS STATEMENT}

Written informed consent was obtained from the individual(s) for publication of any potentially identifiable images or data included in this article.

\section{AUTHOR CONTRIBUTIONS}

$\mathrm{XH}, \mathrm{CW}, \mathrm{LX}$, and XC contributed to conception and design of the study. TM, ZkH, HZ, RZ, JZ, YZ, ZH, YW, and FY contributed to the acquisition, analysis, or interpretation of data for the work. LX and LS wrote the first draft of the manuscript. All authors contributed to the article and approved the submitted version.

\section{FUNDING}

The study was supported by the Sanming Project of Medicine in Shenzhen (No. SZSM202011010).

\section{SUPPLEMENTARY MATERIAL}

The Supplementary Material for this article can be found online at: https://www.frontiersin.org/articles/10.3389/fonc.2021. 585983/full\#supplementary-material

Supplementary Figure 1 | The IGV picture of BRCA2 exon 11 c.1830A >T, p.K944*

7. Waddell N, Pajic M, Patch AM, Chang DK, Kassahn KS, Bailey P, et al. Whole Genomes Redefine the Mutational Landscape of Pancreatic Cancer. Nature (2015) 518(7540):495-501. doi: 10.1038/nature14169

8. Golan T, Hammel P, Reni M, Van Cutsem E, Macarulla T, Hall MJ, et al. Maintenance Olaparib for Germline Brca-Mutated Metastatic Pancreatic Cancer. New Engl J Med (2019) 381(4):317-27. doi: 10.1056/NEJMoa1903387

9. Mehta M, Sinha J, Ogawa M, Ganguly A, Xiang D, Poddar N. Unusual Case of Squamous Cell Carcinoma of Pancreas With Review of Literature. J Gastrointest Cancer (2015) 46(4):426-9. doi: 10.1007/s12029-015-9712-5

10. Ntanasis-Stathopoulos I, Tsilimigras DI, Georgiadou D, Kanavidis P, Riccioni O, Salla C, et al. Squamous Cell Carcinoma of the Pancreas: A Systematic Review and Pooled Survival Analysis. Eur J Cancer (Oxford England: 1990) (2017) 79:193-204. doi: 10.1016/j.ejca.2017.04.006

11. Taibi A, Jacques J, Durand Fontanier S, Charissoux A, Bardet SM, Christou N, et al. Long-Term Survival After Surgery of Pancreatic Primary Squamous Cell Carcinoma: A Case Report and Literature Review. Clin Case Rep (2019) 7 (11):2092-101. doi: 10.1002/ccr3.2429

12. Tella SH, Kommalapati A, Yadav S, Bergquist JR, Truty MJ, Durgin L, et al. Survival and Prognostic Factors in Patients With Pancreatic Squamous Cell Carcinoma. Eur J Surg Oncol: J Eur Soc Surg Oncol Br Assoc Surg Oncol (2019) 45(9):1700-5. doi: 10.1016/j.ejso.2019.05.011 
13. Kumar Das Majumdar S, Kumar Muduly D, Mishra S, Mohapatra CRR, Bunger D, Khan MA. Management of Primary Squamous Cell Carcinoma of the Pancreas With a Nanosomal Paclitaxel Lipid Suspension-Based Regimen: A Case Report. Mol Clin Oncol (2019) 10(4):430-4. doi: 10.3892/mco.2019.1814

14. Schultheis AM, Nguyen GP, Ortmann M, Kruis W, Buttner R, Schildhaus HU, et al. Squamous Cell Carcinoma of the Pancreas in a Patient With Germline Brca2 Mutation-Response to Neoadjuvant Radiochemotherapy. Case Rep Oncol Med (2014) 2014:860532. doi: 10.1155/2014/860532

15. Von Hoff DD, Ramanathan RK, Borad MJ, Laheru DA, Smith LS, Wood TE, et al. Gemcitabine Plus Nab-Paclitaxel is an Active Regimen in Patients With Advanced Pancreatic Cancer: A Phase I/II Trial. J Clin Oncol: Off J Am Soc Clin Oncol (2011) 29(34):4548-54. doi: 10.1200/JCO.2011.36.5742

16. Mohyuddin GR, Aziz M, Britt A, Wade L, Sun W, Baranda J, et al. Similar Response Rates and Survival With PARP Inhibitors for Patients With Solid Tumors Harboring Somatic Versus Germline BRCA Mutations: A Meta- analysis and Systematic Review. BMC Cancer (2020) 20(1):507. doi: 10.1186/ s12885-020-06948-5

Conflict of Interest: The authors declare that the research was conducted in the absence of any commercial or financial relationships that could be construed as a potential conflict of interest.

Copyright (c) 2021 Huang, Wang, Ma, Huang, Zhou, Xu, Zhang, Zhao, Zhang, Huang, Shao, Wang, Yang and Che. This is an open-access article distributed under the terms of the Creative Commons Attribution License (CC BY). The use, distribution or reproduction in other forums is permitted, provided the original author(s) and the copyright owner(s) are credited and that the original publication in this journal is cited, in accordance with accepted academic practice. No use, distribution or reproduction is permitted which does not comply with these terms. 